\title{
Mechanical and Clinical Evaluation of a Shape Memory Alloy and Conventional Struts in a Flexible Scoliotic Brace
}

\author{
Wing-Yu Chan, ${ }^{1}$ Joanne Yip $10,{ }^{1}$ Kit-Lun Yick, ${ }^{1}$ Sun-Pui NG, ${ }^{2}$ Lu Lu, ${ }^{1}$ Kenneth Man-Chee Cheung, ${ }^{3}$ \\ Kenny Yat-Hong Kwan, ${ }^{3}$ Jason Pui-Yin Cheung, ${ }^{3}$ Kelvin Wai-Kwok Yeung, ${ }^{3}$ and Chi-Yung Tse ${ }^{4}$ \\ ${ }^{1}$ Institute of Textiles and Clothing, The Hong Kong Polytechnic University, Hung Hom, Kowloon, Hong Kong SAR, China; \\ ${ }^{2}$ Hong Kong Community College, The Hong Kong Polytechnic University, Kowloon, Hong Kong SAR, China; ${ }^{3}$ Department of \\ Orthopaedics and Traumatology, Li Ka Shing Faculty of Medicine, The University of Hong Kong, Pokfulam, Hong Kong SAR, \\ China; and ${ }^{4}$ Centre for Orthopaedic Surgery, Central, Hong Kong SAR, China
}

(Received 6 January 2018; accepted 23 March 2018; published online 24 April 2018)

Associate Editor Peter E. McHugh oversaw the review of this article.

\begin{abstract}
Smart materials have attracted considerable attention in the medical field. In particular, shape memory alloys (SMAs) are most commonly utilized for their superelasticity (SE) in orthopaedic treatment. In this study, the resin struts of a flexible brace for adolescent idiopathic scoliosis (AIS) are replaced with different conventional materials and an SMA. The corrective mechanism mainly depends on the compressive force applied by the brace at the desired location. Therefore, the mechanical properties of the materials used and the interface pressure are both critical factors that influence the treatment effectiveness. The results indicate that titanium is the most rigid among the five types of materials, whereas the brace with SMA struts presents the best recovery properties and the most stable interface pressure. A radiographic examination of two patients with AIS is then conducted to validate the results, which shows that the SMA struts can provide better correction of thoracic curvature. These findings suggest that SMAs can be applied in orthoses because their SE allows for continuous and controllable corrective forces.
\end{abstract}

Keywords-Nitinol, Bending stiffness, Interface pressure, Flexible brace, Material selection, Scoliosis.

\section{INTRODUCTION}

Scoliosis is the three-dimensional deformity of the spine and trunk. ${ }^{14}$ Adolescent idiopathic scoliosis (AIS) is the most common type of scoliosis, and emerges at or near the beginning of puberty and before skeletal maturity (generally between 10 and 16 years

Address correspondence to Joanne Yip, Institute of Textiles and Clothing, The Hong Kong Polytechnic University, Hung Hom, Kowloon, Hong Kong SAR, China. Electronic mail: tcjyip@polyu. edu.hk old). ${ }^{19}$ Non-surgical treatment, such as immobilization or dynamically correct posture by application of corrective forces with a spinal cast or brace, is an important treatment modality to prevent curve progression and reduce the spinal deformity of AIS patients with moderate scoliosis and a spinal curve that ranges from $20^{\circ}$ to $45^{\circ}$.

Conventional rigid scoliotic orthoses, such as the Milwaukee brace, are fabricated with rigid materials, such as polyethylene and stainless steel, to provide support and stabilize the trunk and spine of patients. ${ }^{4}$ Recently, rigid braces are being constructed with more lightweight materials, such as carbon ${ }^{1}$ and polycarbonate, ${ }^{15}$ to improve their wear comfort. Most of these materials are widely used because of their good mechanical properties, low cost, and acceptable biocompatibility $^{23,24}$; however, they may not be able to satisfy the needs of patients because the rigid materials may cause pain, restrict daily activities and affect selfimage. $^{3}$

To overcome the problems of rigid scoliotic orthoses, new flexible braces composed of textile fabrics and straps have been developed, such as Spinecor, TriaC, Spinealite ${ }^{8}$ and a posture correction girdle. ${ }^{11}$ The researchers claim that these braces have greater wear comfort and provide users with a better self-image and greater mobility in daily life. However, the braces can easily deform, which means that they do not offer optimal corrective effects. ${ }^{21}$ Therefore, smart materials have attracted considerable attention in the medical field as an alternative option and are expected to play a crucial role in the translation of laboratory findings into clinical devices. ${ }^{18}$ Shape memory alloys (SMAs) are an example of a smart material. 
SMAs are most commonly utilized for their superelasticity (SE) in orthopaedic treatment. ${ }^{12} \mathrm{SE}$ is the condition in which the SMA reverts back to its original shape after mechanical loading is applied at temperatures between the austenite finish (Af) temperature and the highest temperature at which martensite transformation can no longer be stress induced $(\mathrm{Md})$ without the need for thermal activation. ${ }^{6}$ SE can also be regarded as the ability to provide continuous and controllable corrective forces that allow orthoses to function accordingly. ${ }^{20}$ Nickel titanium (NiTi) or nitinol is a widely recognized and accepted SMA in the medical industry ${ }^{17}$ because of its biocompatibility. ${ }^{13}$

Corrective forces are a crucial factor for the effectiveness of orthoses, and the amount of corrective force induced is affected by the mechanical properties of the material used. Rigid orthoses can apply corrective forces more consistently while flexible orthoses are more comfortable to wear. However, the relationship between the mechanical properties of a material and the interface pressure that is applied by non-surgical orthopaedic devices is seldom discussed in the current literature. Previous studies have either focused on one type of implant material and its mechanical performance $^{7}$ or measuring the interface pressure ${ }^{2}$ of orthopaedic implants; however, these issues are usually examined separately. Few studies have examined the interface pressure of non-invasive orthopaedic devices constructed with different types of supportive materials. This study is therefore carried out with the goal to address the current knowledge gap by developing and evaluating supportive materials for non-invasive orthopaedic devices and identifying appropriate supportive inserts that can exert optimal corrective forces onto targeted parts of the body.

\section{MATERIALS AND METHODS}

\section{Fabrication of Non-invasive Orthopaedic Device}

The non-invasive orthopaedic device ${ }^{11}$ in this study is a girdle that enables the posture correction of preteen and teenage girls with early scoliosis and fitted with new supportive materials. This girdle improves posture deviation and reduces the progression of the spinal curvature of scoliosis patients by exerting optimal amounts of corrective forces onto targeted areas of the body. Figure 1 is a sketch of the design and highlights the primary materials used for this girdle, which is a flexible brace fabricated with knitted fabrics, and incorporates elastic straps (shoulder and waistband) and resin bones (RBs) as the supportive material. Originally, this girdle was fabricated in accordance with 27 measured places on the body. However, it is now produced in 3 sizes: small, medium and large. Each different sized girdle has 3 zippers to accommodate wearers with different body shapes. The only difference between them is the length of the girdle. The small size is suitable for subjects who are between 150 and $154 \mathrm{~cm}$ in height, the medium size for subjects between 155 and $159 \mathrm{~cm}$ in height and the large size for subjects between 160 and $164 \mathrm{~cm}$ in height. During the fitting session, only the paddings need to be inserted into the pockets in accordance with the radiographic images. A prosthetist confirms the fit of the flexible girdle.

\section{Preparation and Mechanical Characterization}

\section{Preparation of Strut Samples}

The RBs were used as the supportive struts for the orthopaedic device and embedded into the fabric channels that were sewn onto the posture correction girdle. All of these RBs have the same width $(5 \mathrm{~mm})$ but with 3 different thicknesses of $1.3,1.5$ and $1.8 \mathrm{~mm}$. In this study, four other types of materials are used as the struts for comparison purposes: acrylic (ACR), aluminum (AL), titanium (TI) and NiTi (Ti-55 wt \% $\mathrm{Ni}$ ). The size of these struts was determined in accordance with the original RBs used in the girdle. All of the samples were placed in a conditioned room $(23 \pm$ $2{ }^{\circ} \mathrm{C}, 50 \pm 10 \%$ relative humidity) for 40 hours prior to the commencement of testing.

\section{Heat Treatment of SMA}

A binary NiTi SMA that contains approximately 50 at. $\%$ nickel and 50 at. $\%$ titanium was purchased from Jiangyin Fasten-plt Materials Science Co. Ltd., an SMA supplier in Mainland China. The binary NiTi SMA was exposed to heat to create SE. The binary NiTi SMA was treated in an oven at $800{ }^{\circ} \mathrm{C}$ for $1 \mathrm{~h}$ and then cooled down by using a water-quenching method based on the method in Yeung et al. ${ }^{22}$ and Zhou et al. ${ }^{26}$

\section{Bending and Recovery Test}

The strut materials underwent a bending and recovery test which was conducted through a 3-point bending method by using an Instron tensile tester (Instron ${ }^{\circledR}$, Model 4411, U.S.). The span length of the support frame for the test samples is $165 \mathrm{~mm}$, which was determined in accordance with the waistband of the posture correction girdle. After a sample was placed onto the support frame at a right angle to the edges of the supporting points, the test started with the load nose moving downward at a uniform rate of $(5 \pm$ 1) $\mathrm{mm} / \mathrm{min}$. Three bending depths of 5,10 and $15 \mathrm{~mm}$ 

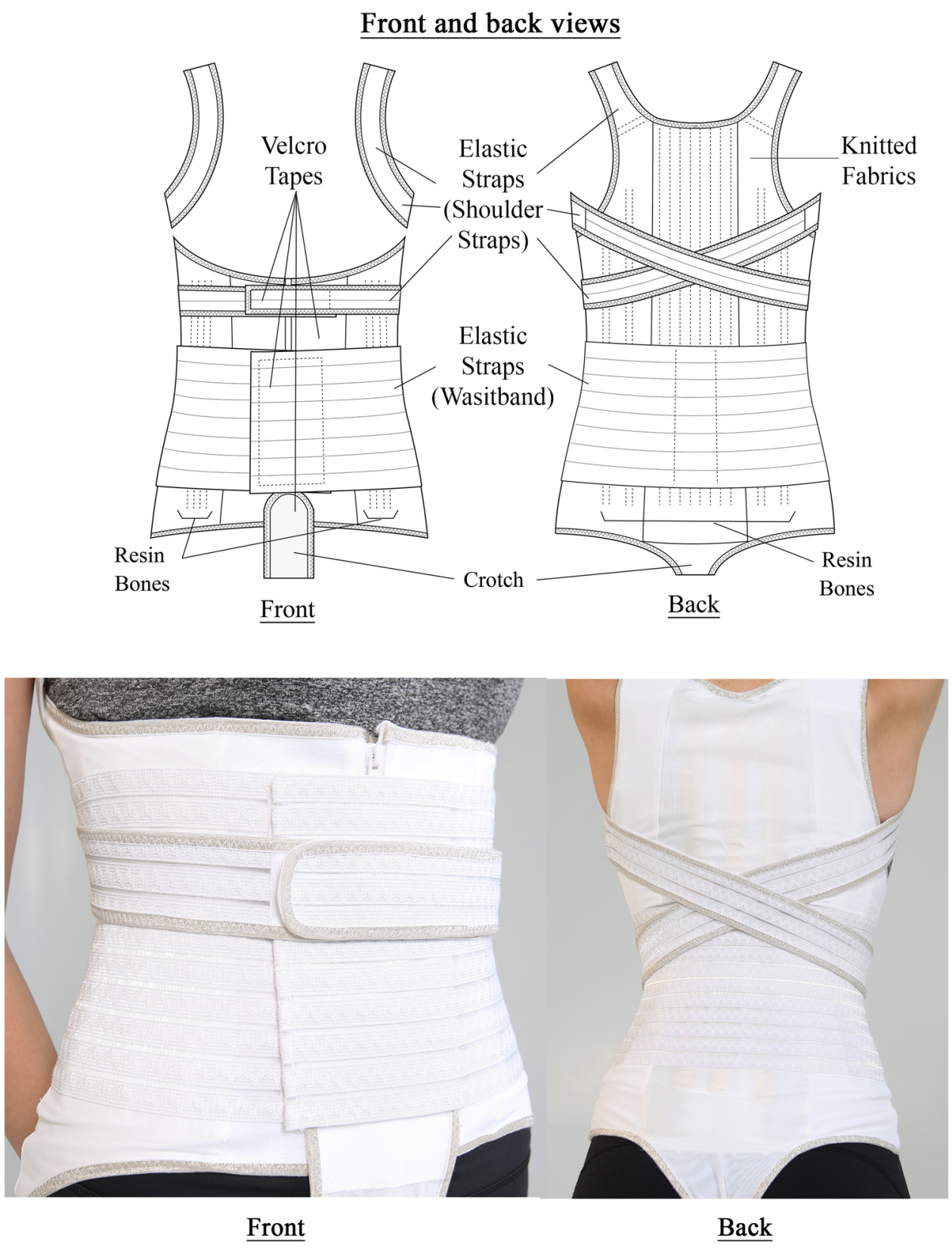

FIGURE 1. Sketch of girdle design and primary materials used to fabricate posture correction girdle.

were tested. Five samples of each type of material were used in the experiment, and the load-deflection curves, stiffness $(\mathrm{N} / \mathrm{mm})$ and recovery $(\%)$ of the different materials were calculated and compared.

\section{Interface Pressure on Soft Mannequin}

In this study, the interface pressure of the posture correction girdle is measured by using a soft mannequin $^{25}$ to reliably compare the different supporting materials under controlled conditions because physiological variations found in a human being, such as body sway, can be minimized accordingly. Since the mannequin and girdle are both symmetrical, the pressure sensors were only placed onto 3 positions of one side of the body or the right side of the underbust, waist and pelvis. The underbust level was determined as the end of the sternum, and the waist level was determined as the middle point between the 10th rib and the pelvis. The Pliance ${ }^{\circledR}-\mathrm{xf}-16$ system with a $3 \times 3$ socket sensor, which provides a sensing area of $30 \times 30$ $\mathrm{mm}^{2}$ and pressure range from 0 to $200 \mathrm{kPa}$, and a Pliance ${ }^{\circledR}$-xf analyser are used in all of the pressure tests because the non-invasive orthopaedic device is similar to a garment and the Pliance X System shows 


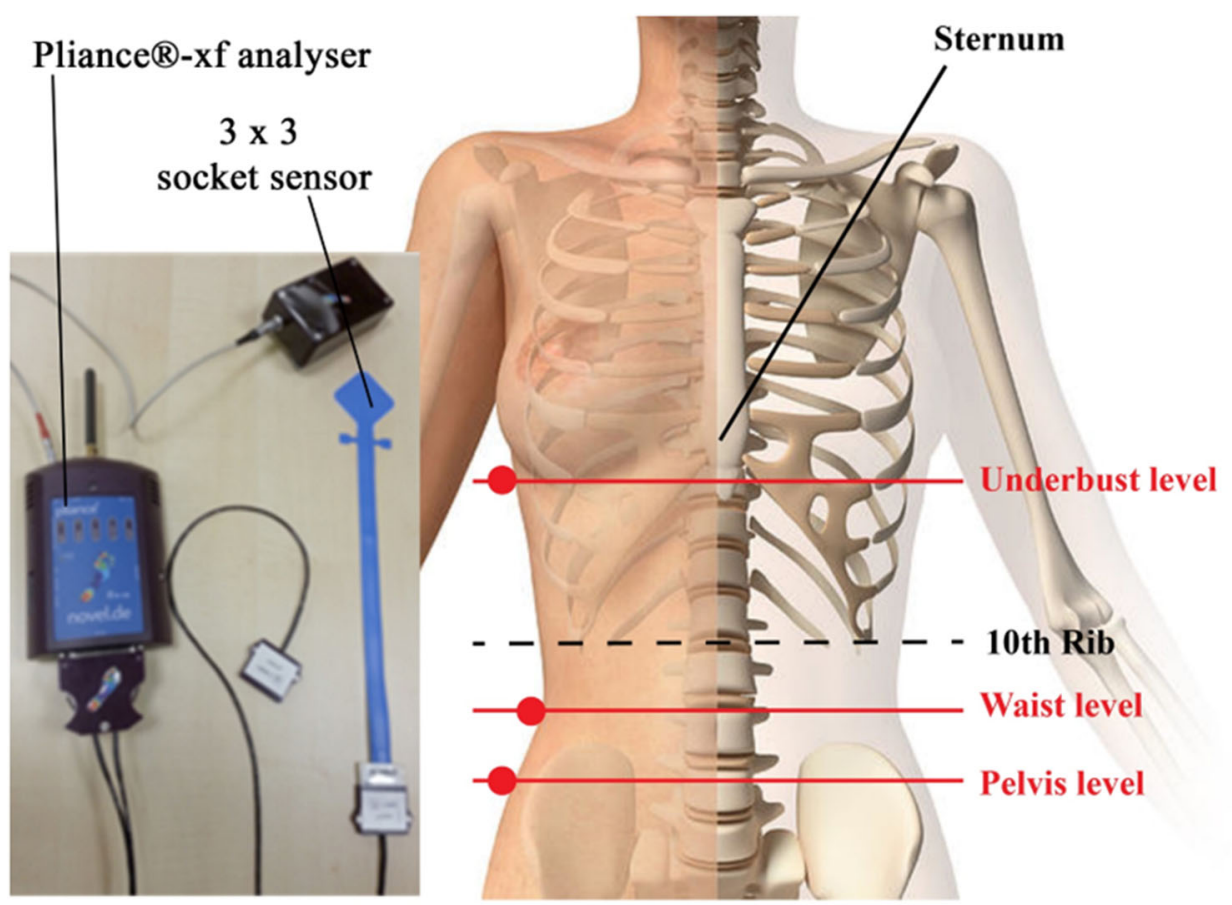

\section{Front view of soft mannequin}

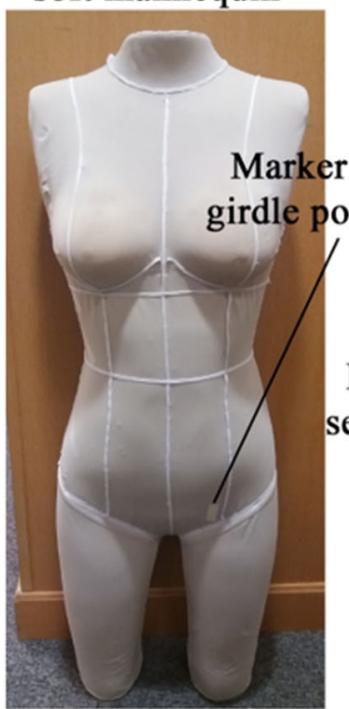

Side view of soft mannequin

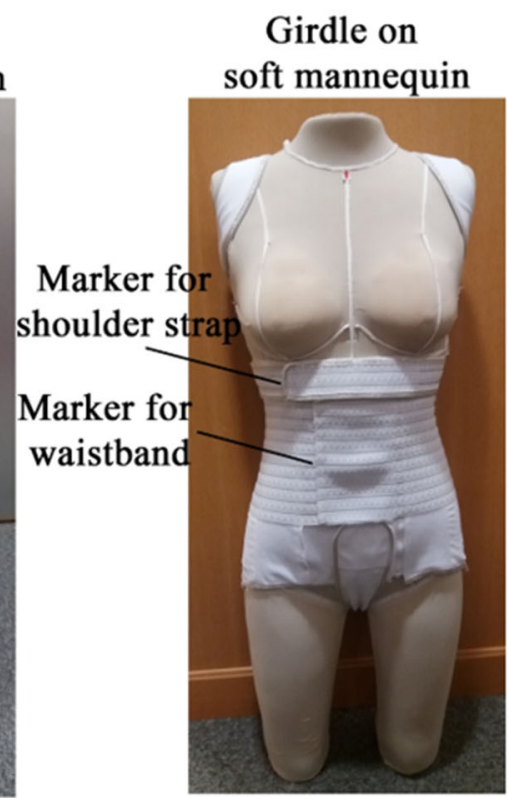

FIGURE 2. Locations of pressure sensors and the girdle on soft mannequin during measurement process.

high test-retest and inter-rater reliability with good linearity when measuring the interface pressure produced by pressure garments. ${ }^{9}$ Each sensor measured the pressure for $5 \mathrm{~s}$. Figure 2 shows the 3 positions of the pressure sensors during the measurement process. The sensor position, stretch length of the elastic band and brace location on the mannequin were marked before the pressure measurement started. The sensor can be temporarily attached onto the mannequin until the pressure measurement is finished. Therefore, the pressure differences due to the usage of the device are eliminated. The girdle-wearing process was repeated three times on the mannequin, and pressure measurements were recorded at the first and third donning. After obtaining the measurements, the mean of the measurements recorded at the 3 locations was calculated for comparison purposes. The pressure changes (in percentage) between the first time and third time that the flexible brace was placed onto the mannequin were calculated. 

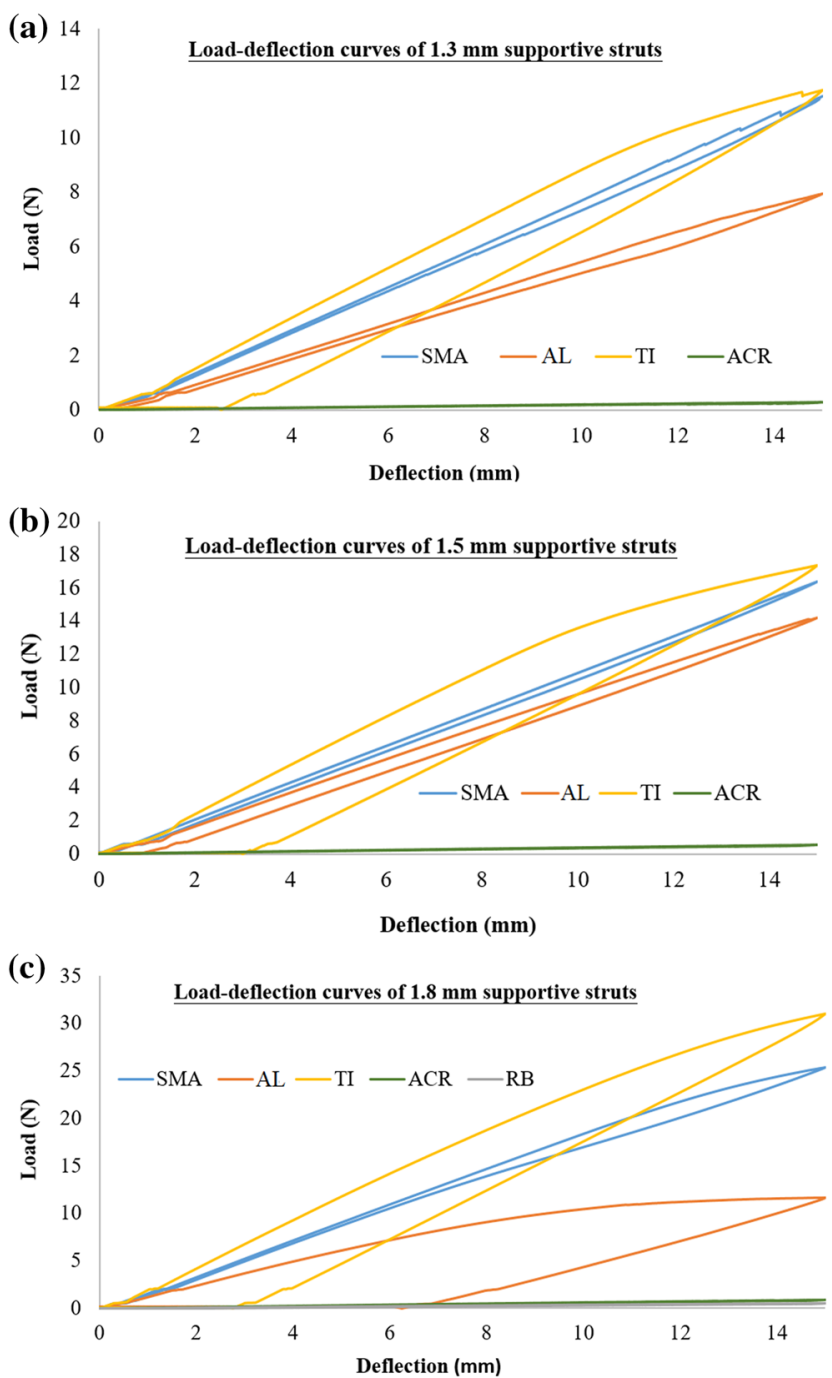

FIGURE 3. Load-deflection curves of different supportive struts at bending depth of $15 \mathrm{~mm}$ (keys: $\mathrm{ACR}=\mathrm{Acrylic}$; SMA= Shape memory alloy; $\mathrm{AL}=$ Aluminum; $\mathrm{Tl}=$ Titanium; $\mathrm{RB}=\mathrm{Resin}$ bone).

TABLE 1. Recovery (\%) of different materials as supportive struts.

\begin{tabular}{lcccrr}
\hline Bending depth & SMA & AL & TI & ACR & RS \\
\hline $5 \mathrm{~mm}$ & $98.92 \pm 0.11$ & $86.64 \pm 5.97$ & $97.16 \pm 0.40$ & $93.94 \pm 0.86$ & $76.61 \pm 2.17$ \\
$10 \mathrm{~mm}$ & $99.06 \pm 0.14$ & $60.13 \pm 0.52$ & $86.60 \pm 0.54$ & $91.63 \pm 0.30$ & $77.53 \pm 0.96$ \\
$15 \mathrm{~mm}$ & $94.53 \pm 0.31$ & $39.54 \pm 3.57$ & $69.90 \pm 2.34$ & $90.17 \pm 0.40$ & $73.76 \pm 1.44$ \\
\hline
\end{tabular}

\section{Statistical Analysis}

The relationship between flexural stiffness and the interface pressure at the level of the underbust, waist or pelvis was analysed by using Microsoft Excel charts. The flexural stiffness of the 4 newly sourced types of struts with 3 different thicknesses was determined from the bending and recovery tests, while the interface pressure of the girdle with the different struts was measured through pressure tests including those for the first donning of the girdle. Scatterplots and polynomial trendlines are used to show the trends in the statistical data by using the $R^{2}$ value.

\section{In Vivo Testing}

\section{Material Selection Criteria}

In the original design of the posture correction girdle, RBs were used as the supportive insertions. Although RBs accommodate body shape well due to their high elasticity, the materials are often too soft to 
establish supportive forces. Also, after donning the brace, the supportive material should maintain its shape, so that supportive forces can be maintained. Therefore, the most suitable supportive insertion material for the flexible brace should have high strength yet good recovery at the same time. High strength means that the struts can help to provide support. Good recovery means that the struts can fixed onto the body over its entire length when the posture correction girdle is worn, and the girdle should be able to recover back to its original shape without deformation. Therefore, both stiffness and recovery properties of the newly selected struts should excel those of the original design.

\section{Recruitment of Scoliotic Subjects}

After the tests were carried out in the laboratory, the most suitable material was selected for use as the

TABLE 2. Flexural stiffness values $(\mathrm{N} / \mathrm{mm})$ of different materials as supportive struts.

\begin{tabular}{lcc}
\hline Sample & Thickness $(\mathrm{mm})$ & Stiffness $(\mathrm{N} / \mathrm{mm})$ \\
\hline \multirow{2}{*}{ SMA } & 1.3 & 0.777 \\
& 1.5 & 1.111 \\
AL & 1.8 & 1.945 \\
& 1.3 & 0.530 \\
& 1.5 & 0.930 \\
TI & 1.8 & 1.503 \\
& 1.3 & 0.913 \\
& 1.5 & 1.485 \\
ACR & 1.8 & 2.723 \\
& 1.3 & 0.021 \\
RB & 1.5 & 0.036 \\
& 1.8 & 0.065 \\
\end{tabular}

new supportive struts of the flexible brace. The effects of the new and original struts were then compared by conducting a wear trial. In this study, the subject selection criteria for both the pressure test and the radiographic examination are as follows: females between the ages of 10 and 14; diagnosis of progressive scoliotic deformity; and a primary Cobb's angle between $25^{\circ}$ and $40^{\circ}$. The study was approved by The Hong Kong Polytechnic University and the Institutional Review Board of the University of Hong Kong/ Hospital Authority Hong Kong West Cluster (HKU/ HA HKW IRB). Informed consent was obtained from the volunteer subjects and their parents.

\section{Radiographic Examination}

Two 14-year-old females participated in the wear trial. They were diagnosed with a Risser sign 4-5 and Scurve spinal deformity, and a Risser sign 1 and C-curve spinal deformity respectively. They had a Cobb's angle of $34^{\circ}$ (T5-T10, apex at T7) $/ 24^{\circ}$ (T12-L4, apex at L2) and $28.9^{\circ}$ (T10-L3, apex at L1) respectively. They weigh 48 and $45.3 \mathrm{~kg}$, are 155 and $162 \mathrm{~cm}$ in height, and have a body mass index (BMI) of 19.9 and 17.3, respectively. Based on the material test results, the subjects were asked to wear the posture correction girdle with either the SMA or RB struts for $2 \mathrm{~h}^{10}$ and then undergo a radiographic examination to evaluate the effectiveness of the girdle when it is donned. Two independent orthopaedic doctors were invited to measure the Cobb's angles of each radiographic image. The inter-observer variability in the Cobb's angle measurements is $\pm 5^{\circ}$. The Cobb's angle measured through X-ray images was compared to that measured without the girdle.

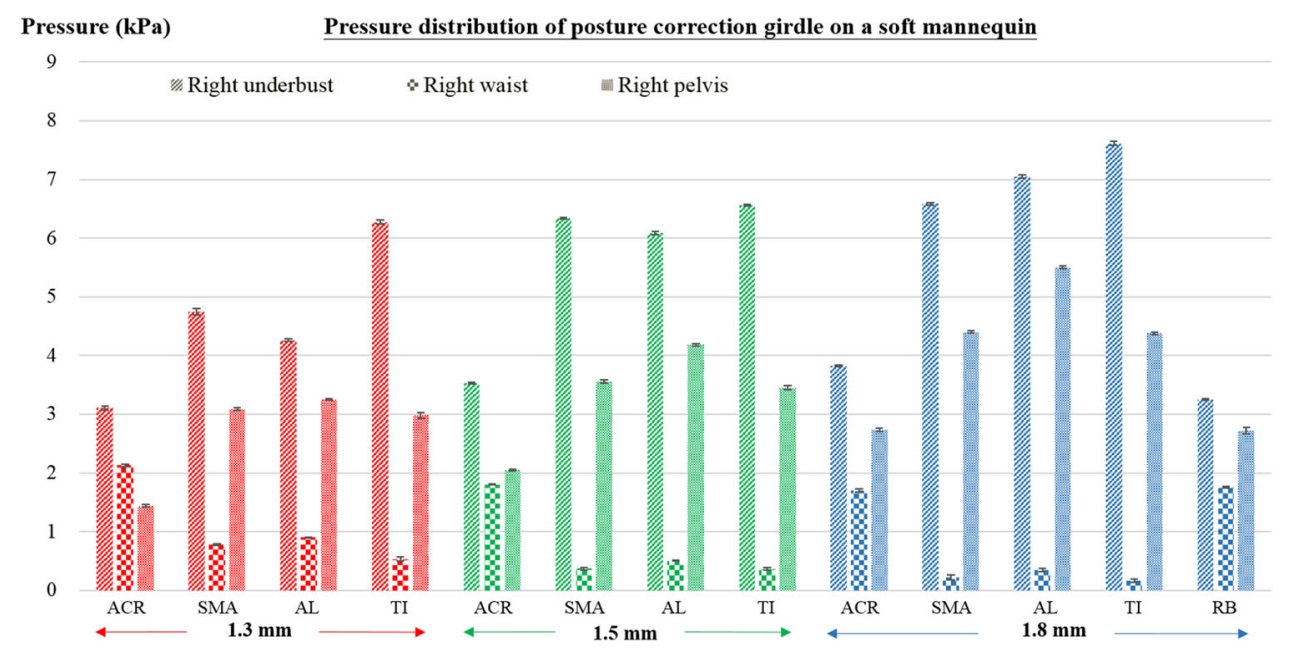

FIGURE 4. Pressure distribution of posture correction girdle with different supportive materials on soft mannequin. 


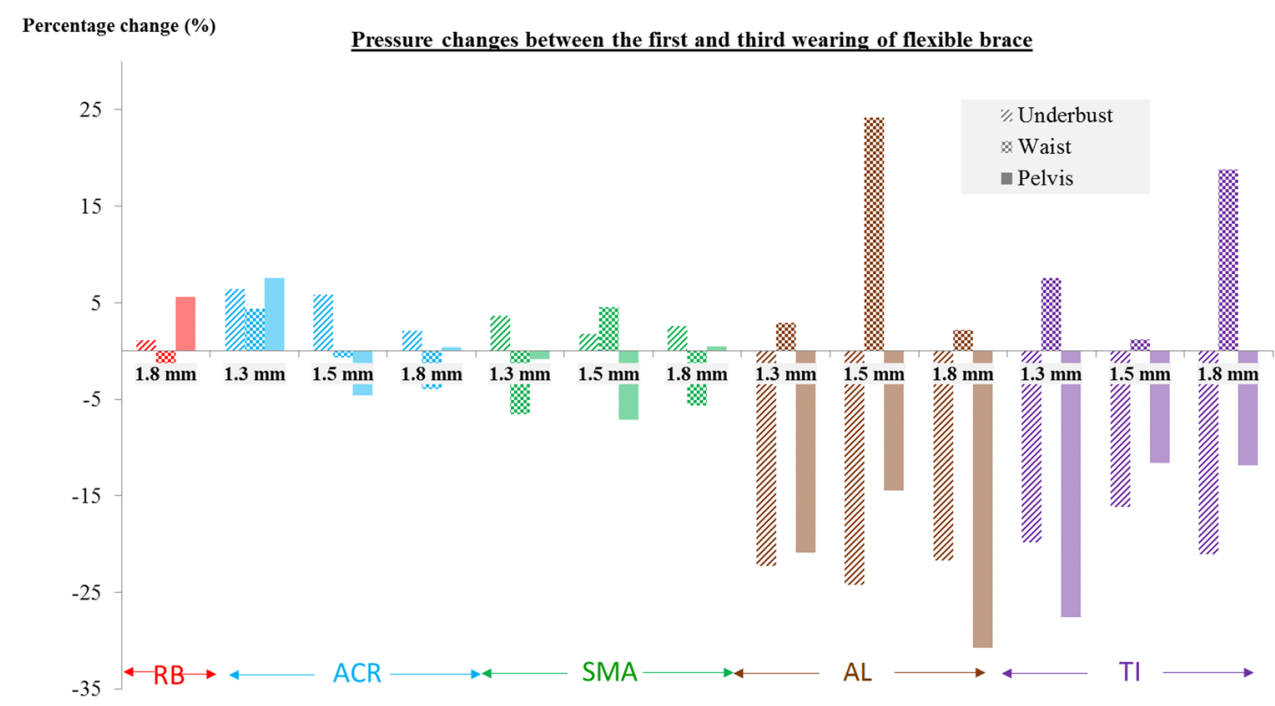

FIGURE 5. Pressure changes between the first and third wearing of posture correction girdle with different supportive struts.

\section{RESULTS}

\section{Bending and Recovery Test}

In this study, the load-deflection curves and recovery $(\%)$ values of all the supportive struts are recorded and shown in Fig. 3 and Table 1. The stiffness $(\mathrm{N} / \mathrm{mm})$ values of the samples are listed in Table 2 . The results indicate that the TI is the most rigid among the five types of materials. The compressive load that the TI struts can withstand at maximum compressive extension is significantly increased from 11.6 to $31.4 \mathrm{~N}$ with increases in the strut thickness from 1.3 to $1.8 \mathrm{~mm}$. The SMA struts can withstand the second highest compressive load which ranges from 11.5 to $25.1 \mathrm{~N}$, whereas the AL bones can withstand a compressive load that ranges from 8 to $11.8 \mathrm{~N}$. Since the ACR struts and RBs are more flexible than the metal struts, they have the lowest bending force. With regard to recovery, the SMA struts can achieve a nearly $100 \%$ recovery, whereas the TI struts showed a reduction in recovery from approximately $97-70 \%$ for bending depths between 5 and $15 \mathrm{~mm}$. The AL struts have poor recovery at only $40 \%$, whereas the plastic materials ACR and RBs have a recovery of approximately 90 and $75 \%$, respectively.

\section{Interface Pressure on Soft Mannequin}

The pressure $(\mathrm{kPa})$ distribution of the posture correction girdle with different supportive materials on the underbust, waist and pelvis areas was recorded on a soft mannequin and is shown in Fig. 4. With regard to the pressure induced onto the underbust, the TI struts with a thickness of $1.8 \mathrm{~mm}$ exert the greatest amount of pressure of $7.6 \mathrm{kPa}$. The AL struts with the same thickness exert the second greatest amount of pressure, followed by the TI struts with a thickness of $1.5 \mathrm{~mm}$ and
SMA struts with a thickness of $1.8 \mathrm{~mm}$. In terms of the plastic materials, the ACR struts and RBs with a thickness of 1.3 and $1.8 \mathrm{~mm}$ apply the least amount of pressure of 3.1 and $3.3 \mathrm{kPa}$, respectively. As for the measured induced pressure onto the pelvis, the $\mathrm{AL}$ struts with a thickness of $1.8 \mathrm{~mm}$ induce the greatest amount of pressure of $5.5 \mathrm{kPa}$, whereas the TI and SMA struts both with a thickness of $1.8 \mathrm{~mm}$ apply a similar amount of pressure of $4.4 \mathrm{kPa}$. The ACR struts with a thickness of 1.3 and $1.5 \mathrm{~mm}$ and $\mathrm{RBs}$ with a thickness of $1.8 \mathrm{~mm}$ apply the least amount of pressure of approximately $1.4,2.1$ and $2.7 \mathrm{kPa}$, respectively. In terms of the pressure induced onto the waist area, the results are entirely different. The ACR struts with a thickness of 1.3 and $1.5 \mathrm{~mm}$ and RBs with a thickness of $1.8 \mathrm{~mm}$ exert the greatest amount of pressure which ranges from 1.8 to $2.1 \mathrm{kPa}$. The TI bones with a thickness of $1.8 \mathrm{~mm}$ exert the least amount of pressure of $0.2 \mathrm{kPa}$.

The changes in the amount of pressure exerted between the first and third donning of the girdle is shown in Fig. 5. The girdle with the AL and TI struts show the greatest changes in pressure. At the level of the underbust, the greatest change of $24.2 \%$ is found with the girdle that has AL struts with a thickness of $1.5 \mathrm{~mm}$, followed by those with a thickness of $1.3 \mathrm{~mm}$ and then a thickness of $1.8 \mathrm{~mm}$. At the level of the waist, the girdles that have AL struts with a thickness of $1.5 \mathrm{~mm}$ and TI struts with a thickness of $1.8 \mathrm{~mm}$ show the greatest change in pressure of approximately 24.2 and $18.8 \%$, respectively. At the level of the pelvis, the girdles with AL and TI struts that have a thickness of 1.8 and $1.3 \mathrm{~mm}$ show a 30.7 and $27.6 \%$ difference in pressure, respectively. Due to small fluctuations in pressure, only minimal differences are found among the ACR, SMA and RB struts 
(a) The relationship between flexural stiffness and pressure on underbust

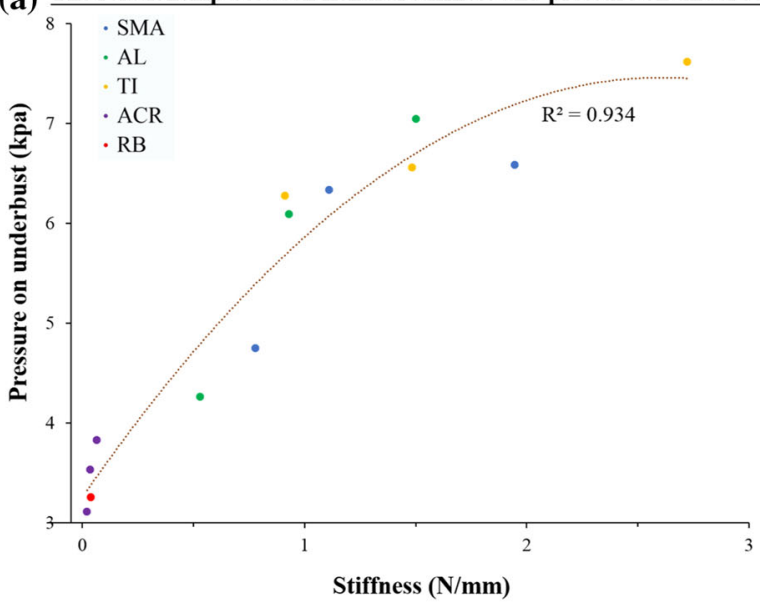

(b) The relationship between flexural stiffness and pressure on waist

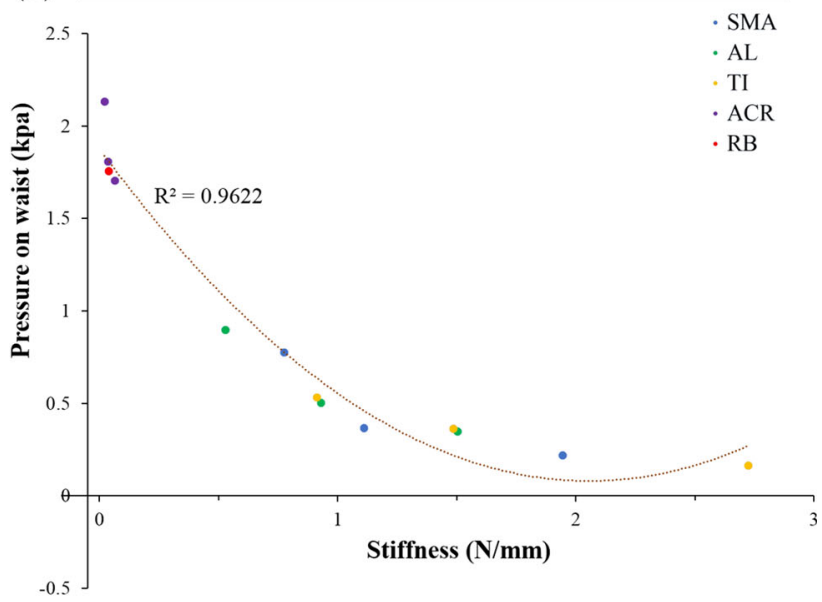

(c) The relationship between flexural stiffness and pressure on pelvis

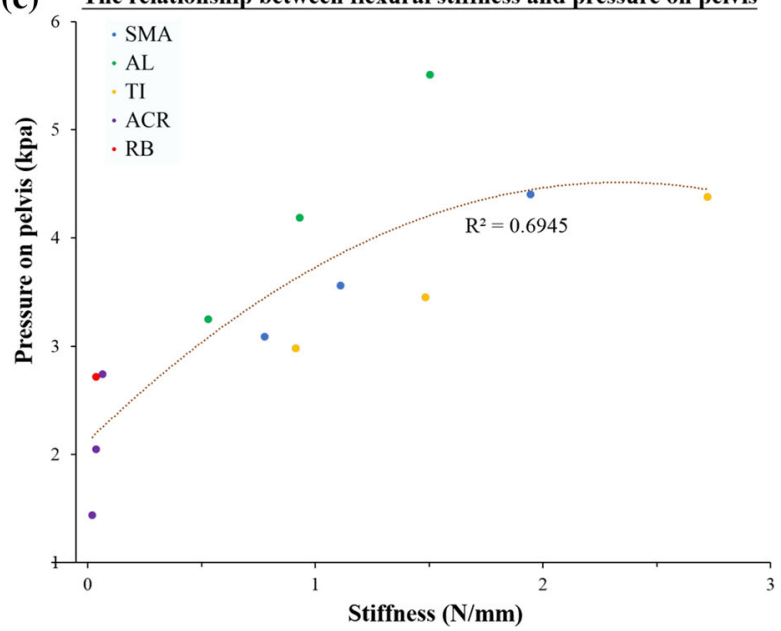

FIGURE 6. Scatterplots with polynomial trendlines of flexural stiffness vs. pressure on different areas.

The data on the pressure in the areas of the underbust, waist or pelvis based on the flexural stiffness of the material are plotted as scatterplots and polynomial trendlines as shown in Fig. 6. Regarding the pressure on the underbust, the order 2 polynomial trendline shows a positive relationship between stiffness and interface pressure. As the stiffness increases, the interface pressure also tends to increase. The figure also shows that the stiffness and interface pressure are highly correlated $\left(R^{2}=0.934\right)$. As for the pressure at the level of the waist, there is a negative relationship between stiffness and interface pressure with an obvious non-linear pattern. As the stiffness increases, the interface pressure tends to decrease. The $R^{2}$ values show that the stiffness and interface pressure are highly correlated $\left(R^{2}=0.962\right)$. With regard to the pressure on the pelvis, there is a positive relationship between stiffness and interface pressure. The $R^{2}$ values show that the stiffness and interface pressure are also correlated $\left(R^{2}=0.695\right)$.

\section{Radiographic Examination}

After the laboratory tests were completed, the SMA struts with a thickness of $1.8 \mathrm{~mm}$ were selected for the preliminary wear trial due to the high pressure performance and maintenance of a proper shape after examining the stiffness and recovery properties of the 5 types of struts. This preliminary wear trial was conducted to determine the effectiveness of the posture correction girdle with SMA and RB supportive struts. Figure 7a shows the X-ray images of the scoliosis patient before and after wearing the flexible brace with SMA struts. Before wearing the girdle, the Cobb's angle of the patient was $34^{\circ}$ in the thoracic region and $24^{\circ}$ in the lumbar region, whereas after wearing the girdle, the Cobb's angles were $22.7^{\circ}$ and $23.8^{\circ}$, respectively. Therefore, the angle of the spinal curve in the thoracic region is reduced by approximately $11.3^{\circ}$ (reduction of $33 \%$ in the Cobb's angle), which is comparable to that through conventional bracing treatment. ${ }^{16}$ Unfortunately, very little improvement was observed for the lumbar curve. With regard to the effectiveness of the flexible brace with RB struts, Fig. 7b shows that before the patient wore the girdle, her Cobb's angle was $28.9^{\circ}$ in the thoracolumbar region, whereas after wearing the girdle, her Cobb's angle is $21.4^{\circ}$. Therefore, the angle of the spinal curve is reduced by approximately $7.5^{\circ}$ (reduction of $26 \%$ in the Cobb's angle). According to Fok et al. ${ }^{5}$ the overall percentage of correction of the posture correction girdle for different Lenke types of curves is around $10-20 \%$.

\section{DISCUSSION}

A higher stiffness value indicates that a stronger force is required to bend the material; thus, the material is more difficult to deform. At the same time, good recovery of the supportive materials for orthoses is 
(a)

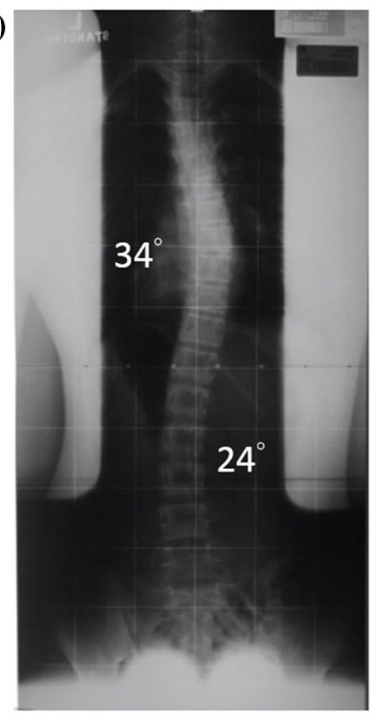

(b)

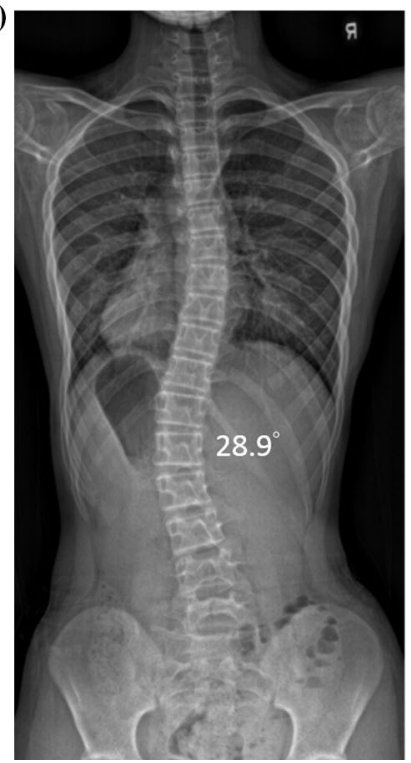

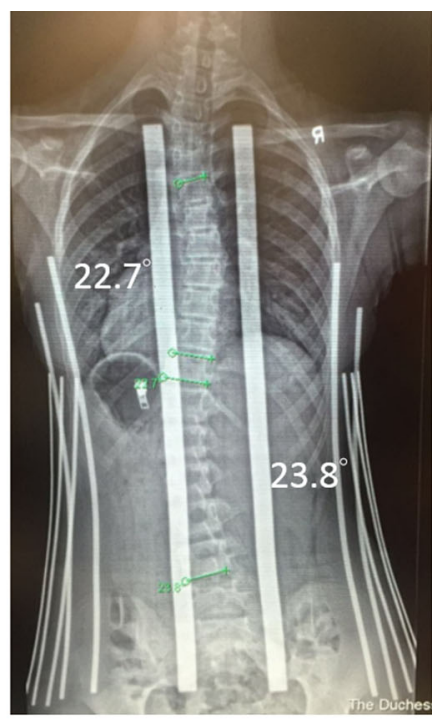

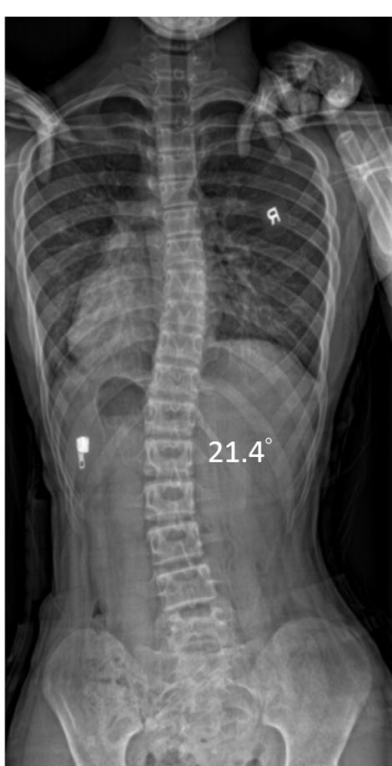

FIGURE 7. X-ray image of scoliosis subject before (left) and after (right) wearing posture correction girdle with (a) SMA supportive struts (b) RB supportive struts.

important because it indicates that the product is durable and allows for the continuous application of pressure/force. Material with more stiffness and good recovery is preferred for the supportive struts of a flexible orthosis because a flexible brace may not be robust enough to apply corrective forces. In addition, the material should be able to maintain its shape after wearing or subjected to daily movement. The bending and recovery results obtained in this study explain why TI has been used in many of the orthoses available today. Although thin TI struts were used, they do not easily deform. Therefore, TI struts are more supportive and lightweight. However, compared to the SMA used in this study, TI is lacking in terms of recovery, and the largest difference in the percentage of recovery between the materials is with a strut thickness of $1.8 \mathrm{~mm}$. Accordingly, the SMA is selected for the posture correction girdle because of its relative high stiffness and excellent recovery properties. Although the TI struts have the highest compressive load, they cannot maintain a good shape after several rounds of bending. Therefore, if TI struts are used on the girdle, they will easily deform, which means that corrective forces cannot be applied continuously. Additionally, overly rigid materials may not be suitable for the flexible brace because compressive forces cannot be applied evenly to the $3 \mathrm{D}$ shape of the human body as demonstrated by the pressure tests.

The pressure tests indicate that more rigid materials apply greater pressure onto the bony parts of the body, 

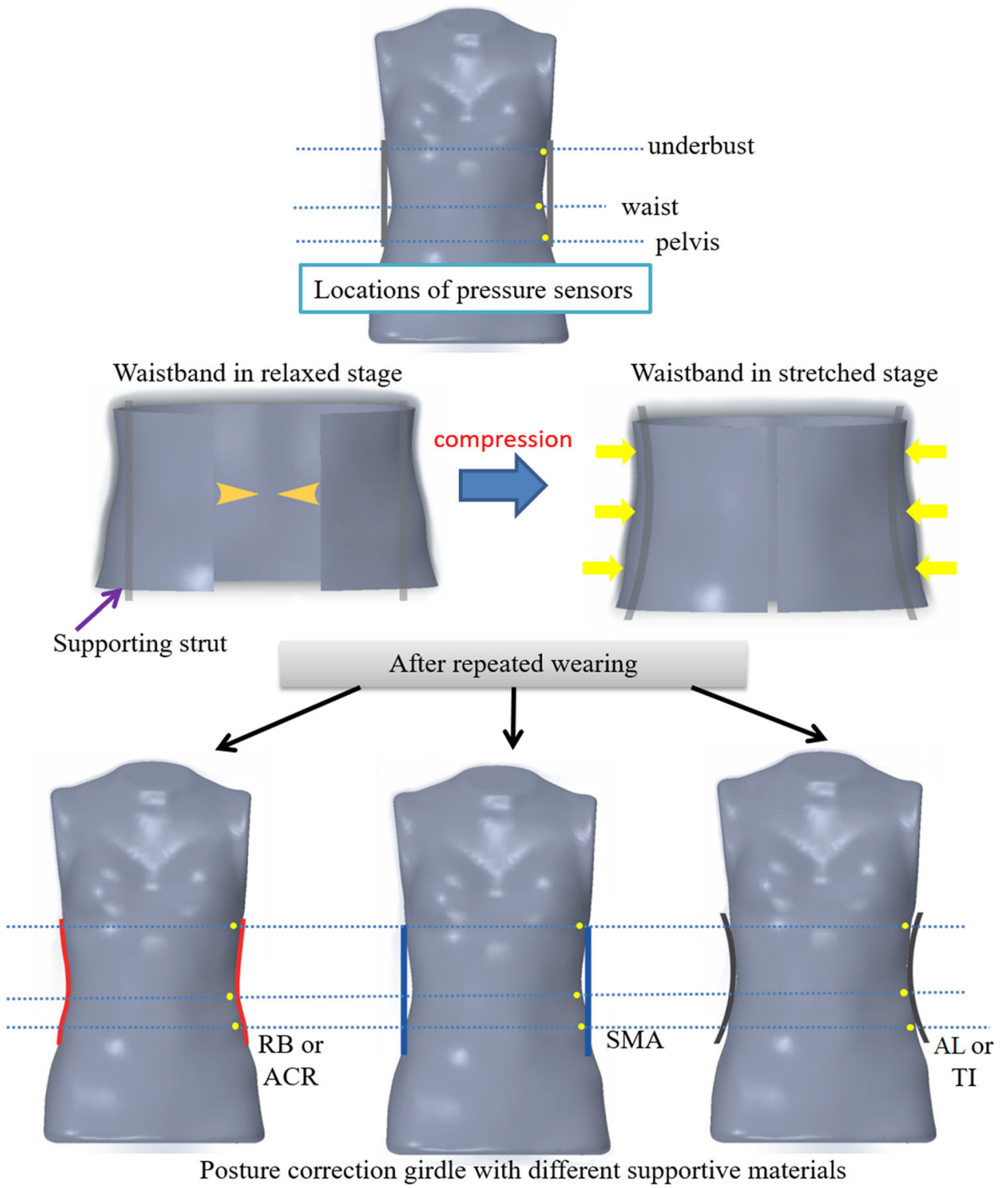

FIGURE 8. The illustration of shape changes of the struts in flexible girdle.

such as the underbust and pelvis areas, while flexible materials apply greater pressure onto less bony areas, such as the waist. Figure 8 shows a schematic diagram to illustrate the findings. Stiffness mostly affects the pressure on the underbust and positively affects the pressure on the underbust and pelvis. However, pressure on the waist is negatively influenced by the material stiffness. In other words, the interface pressure is significantly affected by the contour shape if the material is very stiff. If rigid materials are used in the fabrication of functional apparel, then moulding is recommended to accommodate the body curves before application because of the difficulty in bending rigid materials, which prevents them from accommodating the curves of the body. Rigid materials can only exert high pressure onto convex but not concave body parts.
Although flexible materials cannot exert the same amount of pressure as rigid materials, they can apply force onto both the convex and concave parts of the body. Therefore, when selecting materials for flexible braces, the rigidity of the materials and an even pressure distribution should be taken into consideration.

Moreover, the interface pressure on certain parts of the soft mannequin also changed after repeated wearing of the brace. As shown in Fig. 6, the AL and TI struts show the greatest changes, especially at the underbust and pelvis areas, and these changes are likely caused by the deformation of the struts. The tight elastic waist band of the brace applies force to push the struts against the body, which causes them to bend. After repeated wear, the struts start to curve (as demonstrated in Fig. 8). As a result, the pressure on 
the underbust and pelvis is reduced while that on the waist depends on how the material deforms. If the struts are bent and lean into the most concave point, the pressure on the waist will increase. If they are bent but do not lean into the most concave point, the induced pressure will change slightly. In general, these results agree with the recovery test results. The SMA, ACR and RB materials show good recovery and relatively little change in interface pressure in different positions. Since the SMA material demonstrates SE, the SMA was selected as the new supportive material for the flexible brace after considering the stiffness and recovery properties of all of the different materials for the different struts.

The preliminary wear trial demonstrated a positive relationship between pressure and corrective effects. A comparison of the material test results and pressure data with the radiographies showed that straight SMA struts can induce higher pressure on bony parts (underbust and pelvis) which are probably able to apply a greater amount of force to correct thoracic curvatures. However, these struts cannot easily conform to the body shape; therefore, the compressive forces induced through the girdle onto the waist region may be inadequate, and it is difficult to correct the lumbar spinal deformity. Flexible RB struts can conform to the body so that the deformity in the thoracolumbar region can probably be corrected, although the forces induced onto the bony parts of body are minimal. To improve the effectiveness of thetreatment with the flexible brace, moulded SMA struts are a better option which could conform to the body curves and improve the distribution of pressure over the body itself.

The relationship between material stiffness and corrective forces applied via a flexible brace is determined in this study. Flexible materials can conform to the body shape and induce more even pressure onto different parts of the body. Therefore, these materials may be suitable for the construction of a flexible brace for mild scoliosis cases. With regard to more serious scoliosis cases, materials with greater rigidity, such as AL, TI and SMAs, are more appropriate choices because they can restrict body movement to a greater extent and apply greater amounts of pressure to certain body parts. However, these materials cannot exert pressure evenly because they do not easily bend to conform to the curves of the body. For orthoses that require an even pressure distribution, moulding in accordance with the body shape is recommended. Otherwise, the desired effects of treatment cannot be achieved. Additionally, because of their SE, SMAs have excellent recovery properties and can exert continuous corrective forces. Therefore, the results indicate that SMAs should be used in non-invasive orthopaedic treatments.

\section{LIMITATIONS}

This study is a pilot and therefore the sample size in the in vivo test is small. The SMA struts also require further controlling of their shape. Pre-shaped SMA struts are recommended for a larger clinical trial in the future. On the other hand, each subject only wore one type of brace. It is recommended that they try on two types of girdles for a better comparison. Also, the radiographic images are provided by the subject herself. Although the Cobb's angle measurement is done by the same doctor, different equipment used may also affect the results. Therefore, using different $\mathrm{X}$-ray machines should be avoided when comparing the effect of bracing treatment. In the radiographic examination, it was assumed that the correction obtained through bracing is greater than the potential projection bias.

\section{ACKNOWLEDGMENTS}

The work is supported by funding from the RGC General Research Fund [PolyU 152101/16E] entitled "Anisotropic Textile Braces for Adolescent Idiopathic Scoliosis" and a research studentship granted to Ms. CHAN Wing-yu (RUV9) from The Hong Kong Polytechnic University.

\section{OPEN ACCESS}

This article is distributed under the terms of the Creative Commons Attribution 4.0 International License (http://creativecommons.org/licenses/by/4.0/), which permits unrestricted use, distribution, and reproduction in any medium, provided you give appropriate credit to the original author(s) and the source, provide a link to the Creative Commons license, and indicate if changes were made.

\section{REFERENCES}

\footnotetext{
${ }^{1}$ Bernard, J. C., C. Lecante, J. Deceuninck, G. Notin, L. Journoud, and F. Barral. The carbon brace. Scoliosis. 8:3, 2013.

${ }^{2}$ Courvoisier, A., V. Barbour, W. Skalli, and D. Mitton. In vitro implant-bone interface pressure measurements for a cementless femoral implant, A preliminary study. $J$. Orthop. Sci. 21:487-492, 2016.

${ }^{3}$ Ersen, O., S. Bilgic, K. Koca, T. Ege, E. Oguz, and A. B. Bilekli. Difference between Spinecor brace and Thoracolumbosacral orthosis for deformity correction and quality of life in adolescent idiopathic scoliosis. Acta Orthop. Belg. 82:710-714, 2016.
} 
${ }^{4}$ Fayssoux, R. S., R. H. Cho, and M. J. Herman. A history of bracing for idiopathic scoliosis in North America. Clin. Orthop. Relat. Res. 468:654-664, 2010.

${ }^{5}$ Fok, Q., J. Yip, K. L. Yick, S. P. Ng, and C. Y. Tse. Effectiveness of posture correction girdle as conservative treatment for adolescent idiopathic scoliosis: a preliminary study. Ortho. Res. Online J. 1:1-3, 2018.

${ }^{6}$ Jani, J. M., M. Leary, A. Subic, and M. A. Gibson. A review of shape memory alloy research, applications and opportunities. Mater. Des. 56:1078-1113, 2014.

${ }^{7}$ Kirmanidou, Y., M. Sidira, M. E. Drosou, V. Bennani, A. Bakopoulou, A. Tsouknidas, N. Michailidis, and K. Michalakis. New Ti-alloys and surface modifications to improve the mechanical properties and the biological response to orthopedic and dental implants: a review. Biomed. Res. Int. 1-21:2016, 2016.

${ }^{8}$ Kotwicki, T., J. Durmała, D. Czaprowski, M. Głowacki, M. Kołban, S. Snela, Z. Sliwiński, and I. M. Kowalski. Conservative management of idiopathic scoliosis-guidelines based on SOSORT 2006 Consensus. Ortop Traumatol. Rehabil. 11:379-395, 2009.

${ }^{9}$ Lai, C. H., and C. W. Li-Tsang. Validation of the Pliance $\mathrm{X}$ System in measuring interface pressure generated by pressure garment. Burns. 35:845-851, 2009.

${ }^{10}$ Li, M., M. S. Wong, K. D. Luk, K. W. Wong, and K. M. Cheung. Time-dependent response of scoliotic curvature to orthotic intervention: when should a radiograph be obtained after putting on or taking off a spinal orthosis? Spine. 39:1408-1416, 2014.

${ }^{11}$ Liu, P. Y., J. Yip, K. L. Yick, C. W. M. Yuen, S. P. Ng, C. Y. Tse, and D. Law. An ergonomic flexible girdle design for preteen and teenage girls with early scoliosis. J. Fiber Bioeng. Inform. 7:233-246, 2014.

${ }^{12}$ Machado, L. G., and M. A. Savi. Medical applications of shape memory alloys. Braz. J. Med. Biol. Res. 36:683-691, 2003.

${ }^{13}$ Morgan, N. B. Medical shape memory alloy applications - the market and its products. Sci. Eng. A 378:16-23, 2004.

${ }^{14}$ Negrini, S., A. G. Aulisa, L. Aulisa, A. B. Circo, J. C. de Mauroy, J. Durmala, T. B. Grivas, P. Knott, T. Kotwicki, T. Maruyama, S. Minozzi, J. P. O’Brien, D. Papadopoulos, M. Rigo, C. H. Rivard, M. Romano, J. H. Wynne, M. Villagrasa, H. R. Weiss, and F. Zaina. 2011 SOSORT guidelines: orthopaedic and rehabilitation treatment of idiopathic scoliosis during growth. Scoliosis. 7:3, 2012.
${ }^{15}$ Negrini, S., G. Marchini, and F. Tessadri. "Brace technology" thematic series-The Sforzesco and Sibilla braces, and the SPoRT (Symmetric, Patient oriented, Rigid, Threedimensional, active) concept. Scoliosis. 6:1-18, 2011.

${ }^{16}$ Périé, D., C. E. Aubin, Y. Petit, M. Beauséjour, J. Dansereau, and H. Labelle. Boston brace correction in idiopathic scoliosis: a biomechanical study. Spine. 28:1672-1677, 2003.

${ }^{17}$ Petrini, L., and F. Migliavacca. Biomedical applications of shape memory alloys. J. Metall. 2011:15, 2011.

${ }^{18}$ Shastri, V. P., and A. Lendlein. Materials in regenerative medicine. Adv. Mater. 21:3231-3234, 2009.

${ }^{19}$ Weinstein, S. L., L. A. Dolan, J. C. Cheng, A. Danielsson, and J. A. Morcuende. Adolescent idiopathic scoliosis. The Lancet. 371:1527-1537, 2008.

${ }^{20}$ Wever, D. J., A. G. Veldhuizen, J. De Vries, H. J. Busscher, D. R. A. Uges, and J. R. Van Horn. Electrochemical and surface characterization of a nickel-titanium alloy. Biomaterials. 19:761-769, 1998.

${ }^{21}$ Wong, M. S., J. C. Cheng, T. P. Lam, B. K. Ng, S. W. Sin, S. L. Lee-Shum, H. K. Chow, and S. Y. Tam. The effect of rigid versus flexible spinal orthosis on the clinical efficacy and acceptance of the patients with adolescent idiopathic scoliosis. Spine. 33:1360-1365, 2008.

${ }^{22}$ Yeung, K. W. K., K. M. C. Cheung, W. W. Lu, and C. Y. Chung. Optimization of thermal treatment parameters to alter austenitic phase transition temperature of NiTi alloy for medical implant. Mater. Sci. Eng. A 383:213-218, 2004.

${ }^{23}$ Yeung, K. W. K., R. W. Y. Poon, P. K. Chu, C. Y. Chung, X. Y. Liu, W. W. Lu, D. Chan, S. C. W. Chan, K. D. K. Luk, and K. M. C. Cheung. Surface mechanical properties, corrosion resistance, and cytocompatibility of nitrogen plasma-implanted nickel-titanium alloys: a comparative study with commonly used medical grade materials. $J$. Biomed. Mater. Res. Part A. 82:403-414, 2007.

${ }^{24}$ Yeung, K. W. K., R. W. Y. Poon, X. M. Liu, P. K. Chu, C. Y. Chung, X. Y. Liu, W. W. Lu, D. Chan, S. C. W. Chan, K. D. K. Luk, and K. M. C. Cheung. Nitrogen plasmaimplanted nickel titanium alloys for orthopedic use. Surf. Coat. Technol. 201:5607-5612, 2007.

${ }^{25} \mathrm{Yu}$, W., J. T. Fan, and X. M. Qian. A soft mannequin for the evaluation of pressure garments on human body. Sen'i Gakkaishi. 60:57-64, 2004.

${ }^{26}$ Zhou, D., Y. Gao, M. Lai, H. Li, B. Yuan, and M. Zhu. Fabrication of NiTi shape memory alloys with graded porosity to imitate human long-bone structure. J. Bionic Eng. 12:575-582, 2015. 